\title{
Parts as Counterparts
}

\author{
A. J. COTNOIR
}

WORD COUNT: 4296

\begin{abstract}
Mereological nihilists are faced with a difficult challenge: explaining ordinary talk about material objects. Popular paraphrase strategies involve plurals, arrangements of particles, or fictions. In this paper, a new paraphrase strategy is put forward that has distinct advantages over its rivals: it is compatible with gunk and emergent properties of macro-objects. The only assumption is a commitment to a liberal view of the nature of simples; the nihilist must be willing to accept the possibility of heterogeneous extended simples. The author suggests reinterpreting the parthood and composition relations as modal. According to this paraphrase, composition is a kind of counterpart relation. The author shows that one can accept that mereological nihilism is metaphysically necessary, while endorsing all the claims of classical mereology. As a result, the nihilists are in exactly the same position as the classical mereologist when it comes to explaining talk about ordinary objects, but without the additional ontology.
\end{abstract}

KEYWORDs: mereology, nihilism, paraphrase, composition, simples, gunk, parts, counterparts, emergent properties. 
Some philosophers do not believe in composition. Th They ascribe to mereological nihilism, the view that composition never occurs. But our ordinary way of speaking about the world is rife with talk of composite objects. Typically nihilists attempt to show how ordinary language about composite objects can be paraphrased in a more strict or fundamental language without composites. But it is not enough to merely mimic ordinary object talk; we frequently talk about objects being composed of other objects, of objects being parts of other objects. Nihilists have a burden to paraphrase mereological talk in a way that preserves its utility to ordinary speakers.

The aim of this paper is to provide a paraphrase strategy for the mereological nihilist that achieves both: to mimic talk of ordinary objects and their mereological relations. I do this by borrowing techniques originally developed in literature on persistence and the problem of change. Broadly speaking, the approach exploits a rough analogy between modality and temporality. Nihilists ought to reinterpret mereology as involving modal claims — claims relating to objects in different worlds - while maintaining that at no world does composition occur. On this view, composition and parthood are reduced to counterpart relations. The result, I argue, is that mereological nihilists can, without appeal to composites, find a way of saying everything the classical mereologist can say. This approach has marked advantages over rival paraphrase strategies: it can handle cases involving 'gunk' and 'emergent' properties of objects.

In $\S_{I}$, I lay the groundwork for the paraphrase strategy, detailing some background suppositions involving extended simples. In $\$ 2$, I present a way of interpreting mereological relations as modal claims involving objects at 'duplicate' worlds. In $\S_{3}$, I show that this interpretation satisfies the axioms of classical mereology and explain how the approach can be modified to allow for gunk. In $\$_{4}$, I show how to extend this paraphrase to talk about ordinary material objects and modify it to allow for emergent properties of objects.

\footnotetext{
${ }^{1}$ Wheeler [30], Unger [26], Rosen and Dorr [4] , Cameron [I] , and recently Sider [19]. Partial nihilists include van Inwagen [29], Merricks [13].
} 


\section{Nihilism and Extended Simples}

Let us say that an object is simple iff it has no proper parts. Atomism is the view that everything is simple; and mereological nihilists are atomists.

Simples are generally assumed to be small; they are located in the smallest possible regions of space. They might be point-sized (or planck-sized) particles of microphysics. The thought that simples are small is so natural philosophers often presuppose it without comment.|2

But recently the smallness of simples has come under attack. Some have argued for the possibility of extended simples. [n Indeed, some nihilists believe the world to be made up of only one universe-sized simple or perhaps many ordinary-object-sized simples. These debates crucially depend on the relation between mereology and location. From a purely mereological perspective, the essential feature of simples is that they have no proper parts — not how big or small they are.

Among those who accept the possibility of extended simples, there is further disagreement over the possibility of heterogeneous extended simples — simples that qualitatively differ from themselves (perhaps along their temporal or spatial axes). I I do not wish to weigh in on either of the above debates here. For the purposes of this paper, I simply assume the possibility of heterogeneous extended simples. I wish to show that nihilists who accept the possibility of heterogeneous extended simples have an added advantage: they have a straightforward way of paraphrasing talk of ordinary material objects and their mereological relations.

${ }^{2}$ Perhaps the guiding thought is this: suppose an object $o$ were to occupy an extended (non-point-sized) region of space $R$. But since $R$ is extended, it has some proper subregion $R^{\prime}$. But since $o$ occupies $R$, it must also (partly) occupy $R^{\prime}$; it does so by virtue of having a proper part $o^{\prime}$ that fully occupies $R^{\prime}$. Thus $o$ is not simple.

Of course, this line of argument for the smallness of simples relies on an implicit appeal to DOCTRINE OF ARBITRARY UNDETACHED PARTS, something which is independently controversial (see van Inwagen [28]). But even philosophers sensitive to this controversy often slide freely between simplicity and smallness. Consider van Inwagen [29]:

If this answer [nihilism] is correct, then (if the current physics is to be believed) the physical world consists entirely of quarks, leptons, and bosons - there is just nothing else in it for these particles have no parts and they never add up to anything bigger. (72)

${ }^{3}$ See Markosian [9] and McDaniel [II] . See also Simons [22].

${ }^{4}$ Schaffer [I7] and Rea [I6] are good examples of existence monists. See also Markosian [Io] the emergent nibilism of Williams [3I].

${ }^{5} \mathrm{McDaniel}[\mathrm{I2}]$ and Parsons [I5] both accept them. Spencer [23] argues against. 
Before moving on, however, there is a basic problem with heterogeneous extended simples that deserves consideration. Assuming the indiscernibility of identicals, how could something differ from itself without contradiction?

Fortunately, there are a number of different proposals already in the literature, many of which were originally developed in the literature on persistence. The first proposal is due to Hawthorne and Cortens [I4]. They suggest what they call 'feature-placing' language according to which properties may be 'placed' without requiring a subject (for example: "It is raining here"). 9 A second approach is related to adverbial accounts of instantiation, where the instantiation of a property is relative to a given region.] A third approach is due to Sider [2I] who gives an account of located properties using 'statespace structures'. [ A fourth approach due to Parsons [ [ I5] suggests there are fundamental 'distributional properties' like being polka-dotted.

A final approach due to Ehring [5] (and extended by McDaniel [I [2]) was developed as a solution to Lewis's problem of temporary intrinsics; it involves relativising properties to regions of spacetime. This is the approach to be used in what follows (although it may well be that any of the above approaches could be used). The crux of Ehring and McDaniel's proposal is to appeal to short-lived and highly localised tropes which are instantaneous and unextended. We can classify these tropes by their resemblance to other tropes in the typical nominalist way. Here are the conditions under which objects instantiate properties at times and at regions.

temporal instantiation $x$ is $F$ at $t$ iff $x$ exemplifies an $F$-trope existing at time $t$.

regional instantiation $x$ is $F$ at $r$ iff $x$ exemplifies an $F$-trope existing at $r$.

To see how these clauses work, consider a half-red and half-blue object $x$.

\footnotetext{
${ }^{6}$ See also the developments in Turner [25].

${ }^{7}$ See Lewis [8] for criticisms of this proposal as adopted by endurantists.

${ }^{8}$ Sider develops the approach for a special case of heterogeneous extended simples — the world of the monist but the approach might be adapted more generally.
} 


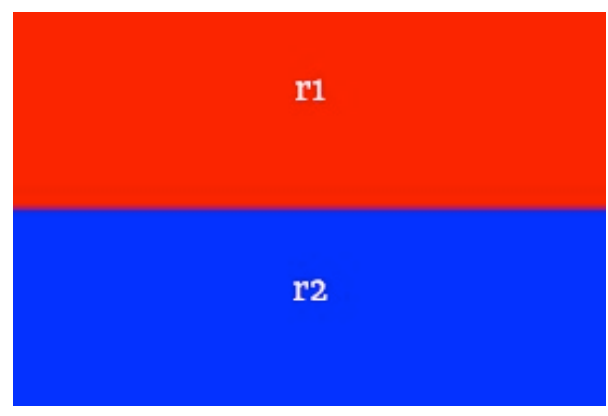

Now, by hypothesis, $x$ is a simple wholly located at the region $r_{1} \cup r_{2}$. Yet, $x$ is red at $r_{1}$ and $x$ is blue at $r_{2}$ by virtue of exemplifying red tropes in $r_{1}$ and blue tropes in $r_{2}$. That is, $x$ need not have proper parts exactly occupying $r_{1}$ or $r_{2}$ for it to differ from itself. All this is, of course, consistent with the indiscernibility of identicals, since $x=y$ even if $x$ is red at $r_{1}$ and $y$ is blue at $r_{2}$. On the EhringMcDaniel proposal, qualitative heterogeneity across space consists in exemplifying non-resembling localised tropes at different spatial regions.

\section{The Paraphrase Strategy}

Mereological relations are usually interpreted as extensional: as holding between objects at a world. I propose that nihilists should interpret mereological relations as intensional: as holding between objects in different worlds.

The application to mereology is new, but the general strategy should be familiar. Sider's [20] stage-theoretic approach to the problem of persistence does away with the need for a temporal parts ontology by employing counterpart relations between stages at different times. If By analogy, this approach does away with the need for a material parts ontology by employing counterpart relations between objects at different worlds.

I begin with an example to guide intuitions before setting out the proposal in detail. Imagine a world $w$ inhabited by only one heterogeneous extended simple: a table. Because the table is simple, there are no other parts in that world, and in particular no subatomic particles. Now imagine

\footnotetext{
${ }^{9}$ Of course, Sider accepts (or, at least did at the time) the temporal parts ontology, but for other reasons. Stage theory does not require it.
} 
another world $w^{\prime}$ with a number of simple subatomic particles arranged in such a way as to appear exactly like the table in $w$. To a first approximation, $w$ and $w^{\prime}$ have a lot in common; indeed, the distribution of properties across spacetime is the same. In one sense (to be specified shortly) $w$ and $w^{\prime}$ are duplicate worlds. Both worlds are nihilistically acceptable, since they contain only simples. But it is also natural to think that the relationship between the particles in $w^{\prime}$ and the table in $w$ is significantly like the ordinary notions of parthood and composition. This is the core idea behind the paraphrase strategy to be explored in below.

So what, exactly, is in common between our table world $w$ and the particle world $w^{\prime}$ ? Here, the Ehring-McDaniel proposal can help. Let us represent the commonalities between these two worlds with point-property arrays, or 'P-arrays' for short.

p-array $A$ is a $P$-array iff $A$ is a non-empty set of spacetime points with a specific distribution of instantaneous localised tropes.

You should think of a P-array as modelling the external world as it exists abstracting away from what things there are. They should not to be thought of as worlds, as worlds are full of material objects. To use a common metaphor, worlds have 'joints'; they have objective structure. This additional structure can be modelled via partitions on P-arrays. 10

partition A set $P$ of non-empty sets is a partition of $A$ if: (i) $P$ covers all of $A$ (i.e. $\cup P=A$ ); and (ii) the members of $P$ are pairwise disjoint (i.e. $p_{i} \cap p_{j}=\emptyset$ for any $p_{i}$ and $p_{j}$ in $P$ s.t. $i \neq j$ ). A partition 'carves up' a P-array into disjoint regions. Partitioning a P-array is a way of locating objects within it. Members of a given partition — 'blocks' — are regions occupied by material objects.

Condition (i) guarantees that in any partition, every point in a P-array is included in some block and hence, every region of spacetime is covered. Without (i), we would be modelling portions of worlds, or merely some but not all of the objects that exist at a world. Condition (ii) rules out the

\footnotetext{
${ }^{10}$ That P-arrays are carved up via partitions is importantly related to Schaffer's [I7] tiling constraint.
} 
same point featuring in more than one block. Without (ii), distinct objects would be allowed to reside in overlapping regions of spacetime. But nihilists typically reject the existence of overlapping objects. [1]

Furthermore, (i) and (ii) are the only conditions to be imposed on worldly structure. That is, I will assume a basic principle of plenitude principle about worlds:

world If $A$ is a P-array, $P$ any partition on $A$, and $D$ a domain of objects located at regions in $P$, then the triple $w=\langle A, P, D\rangle$ is a world.

Every possible partition on every possible P-array represents a possible world. This codifies the thought that the world could have been individuated differently than it is — it might have had a different structure than it has. We might have lived in a monistic world, with the very same point-trope distributions. This possible world is given by the coarsest partition on our P-array $A$ with a single-object domain. Or, we might have lived in a pointy atomistic world along the lines of traditional atomists, represented by the finest partition on our P-array. Or it might have been that the world is individuated into a domain of middle-sized objects like tables and chairs, represented by a mid-grade partition.

It is worth emphasising that these worlds are guaranteed to be nihilistically acceptable. The fact that worlds are modelled by partitions implies that if $r_{1}$ and $r_{2}$ are members of the same partition, then $r_{1}$ is not a proper subregion of $r_{2}$, nor is $r_{2}$ a proper subregion of $r_{1}$. As we will see below, this constraint is important to guarantee that at no world is anything a proper part of anything else; that is, it ensures that at no world does composition occur. [12

It is clear that worlds may be have different objects occupying different regions but be otherwise

\footnotetext{
${ }^{11}$ It is natural to think that objects which overlap spatially overlap mereologically. This is further explored in Schaffer's [ [7] defense of the tiling constraint. But see Williams [3I] for a nihilistic view which allows for completely coincident material objects. It is unclear whether the paraphrase strategy below could be adapted to handle cases of coincidence. One possibility is to use 'divisions' induced by similarity relations instead of partitions a là Hazen and Humberstone [6].

${ }^{12}$ Various people (including an anonymous referee) have asked whether the existence of regions and subregions makes for something like a parthood relation that would be unacceptable to the nihilist. I don't think it does, since we needn't be committed to the literal existence of regions; the paraphrase strategy could easily function using only pluralities of space-time points rather than their regions together with the standard plural-logical 'is one of' relation.
} 
indistinguishable. These worlds exhibit the very same pattern of distribution of tropes across points in spacetime - they are 'duplicates'.

p-duplicate $w_{i}$ and $w_{j}$ are $P$-duplicates iff they are partitions on the same P-array.

But just as worlds can be P-duplicates, so too can objects in those worlds be counterparts.

Let the denotation of a singular term $x$ at world $w\left(\right.$ written $\dot{x}_{w}$ ) be a member of the domain of objects $D$. The location of an object denoted by a singular term $x$ at world $w$ (written $|x|_{w}$ ) is a region of the P-array for $w$. For plural terms, the denotation of $x x$ at world $w$ (written $\dot{x} x_{w}$ ) is a subset of domain $D$. Moreover, the location of an object denoted by a plural term $x x$ at a world $w$ (written $|x x|_{w}$ ) is a collection of regions in the P-array for $w$.

We are now able to define P-counterparthood.

p-counterpart $\dot{x} x_{w_{i}}$ and $\dot{y} y_{w_{j}}$ are P-counterparts iff $w_{i}$ and $w_{j}$ are P-duplicates, and $\bigcup|x x|_{w_{i}}=$ $\bigcup|y y|_{w_{j}}$.

Roughly, some objects are P-counterparts of some other objects whenever they (collectively) occupy the same region of the same P-array. The idea behind this notion of counterparthood is that objects located at the same region of a given P-array have the very same trope distributions, and hence inherit similar properties. Going back to our simple table world and the particle world: these worlds are qualitatively indistinguishable even if the occupants of that world are different.

Ordinarily, counterparthood is a binary relation between single objects. The P-counterpart relation, however, is multigrade: a binary relation between either single objects or pluralities of objects. The simple table and the particles are an example of one-many counterpart relation.13

\footnotetext{
${ }^{13}$ An anonymous referee points to another proposal — the DOT THEORY from Williams [32, \$2.3] (Williams cites Schwarz [I8] as the original source of the idea). The DOT THEORY exploits counterpart-theoretic tools to give a paraphrase strategy for "gavagai" involving undetached rabbit parts. A main difference is that the DOT THEORY involves a number of primitive binary counterpart relations that hold between two things if e.g. they are parts of the same rabbit. This severs the connection between counterpart relations and qualitative similarity. On my proposal, there is a single multigrade counterpart relation that is reducible to qualitative similarity between space-time regions (as above), and hence we do not need any counterpart-theoretic primitives. Still the DOT THEORY is interesting, and worthy of more consideration than I can give it here.
} 
With the notions of P-duplication and P-counterparthood on board, we can proceed to interpret mereological talk. First, consider sentences involving the parthood relation. The following paraphrase can be constructed:

part ' $x$ is part of $y$ ' is correct at $w_{0}$ iff there are worlds $w_{1}$ and $w_{2}$ such that they are P-duplicates of $w_{0}$, and $|x|_{w_{1}} \subseteq|y|_{w_{2}}$.

So, nihilists can paraphrase parthood claims as claims involving objects at different worlds: it is correct to say that an object is part of another just if it occupies a subregion of a world duplicate to our own.

Secondly, proper parthood and overlap can then be paraphrased in straightforward ways:

proper part ' $x$ is a proper part of $y$ ' is correct at $w_{0}$ iff there are worlds $w_{1}$ and $w_{2}$ such that they are P-duplicates of $w_{0}$, and $|x|_{w_{1}} \subseteq|y|_{w_{2}}$ and $|x|_{w_{1}} \neq|y|_{w_{2}}$.

overlap ' $x$ overlaps $y$ ' is true at $w_{0}$ iff there are worlds $w_{1}$ and $w_{2}$ such that they are P-duplicates of $w_{0}$, and the intersection $|x|_{w_{1}} \cap|y|_{w_{2}}$ is non-empty.

Notice that proper parthood is always relation between objects at different worlds. This is a consequence of properties of partitions; at no world are there objects occupying subregions of regions occupied by other objects, as this would violate clause (ii) of PARTITIONs above. It follows, then, that objects are always and everywhere simple in the sense that, strictly and literally speaking, they have no proper parts. For similar reasons, mereological overlap is a transworld relation when it is non-trivial — in cases where two distinct objects overlap. If

Lastly, to paraphrase the composition relation we utilise plural variables.

compose ' $x x$ compose $y$ ' is correct at $w_{0}$ iff there are worlds $w_{1}$ and $w_{2}$ such that they are Pduplicates of $w_{0}$, and $\dot{x} x_{w_{1}}$ and $\dot{y}_{w_{2}}$ are P-counterparts.

\footnotetext{
${ }^{14}$ It may not be immediately obvious from the clause above that overlap satisfies the typical mereological definition: $x$ overlaps $y$ off there is some $z$ that is part of $x$ and part of $y$. One can see, however, that the above paraphrase implies that there is a non-empty region where both objects are (partially) located. By the plenitude principle on worlds, there is some P-duplicate world to $w_{0}$ in which that region is occupied by a single object $\dot{z}$. And from this, it follows that ' $z$ is part of $x$ ' and ' $z$ is part of $y$ ' are both correct.
} 
Composition then is P-counterparthood. It is correct to say that some things compose another whenever the former objects collectively occupy the same region as the latter at duplicate worlds. The only time composition holds within a world is the trivial case of an object composing itself. Non-trivial composition is always transworld; as such, mereological nihilism is necessarily true.

\section{Recovering Classical Mereology (Including Gunk!)}

It remains to be shown that the above definitions and corresponding paraphrases can recover the full power of classical mereology. Before proving the result in full generality, I will run through a basic example to show how the above proposal generates the required mereological structure; after which those readers uninterested in following the proofs may skip them.

For our example, consider the three-element model of classical mereology below. Upward arrows indicate proper parthood. As proper parthood is transitive, if an object may be reached by a sequence of arrows from another object, then the latter is a proper part of the former.

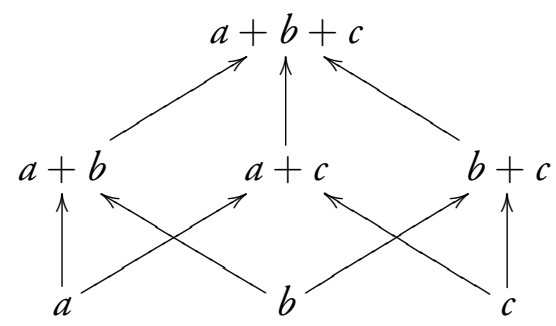

For simplicity, we will assume that $a, b$, and $c$ are all point-sized, occupying the points $\hat{a}, \hat{b}$, and $\hat{c}$ respectively. In this case, our P-array would be $A=\{\hat{a}, \hat{b}, \hat{c}\}$ and $|a|_{w_{0}}=\{\hat{a}\},|b|_{w_{0}}=\{\hat{b}\}$, and $|c|_{w_{0}}=\{\hat{c}\}$. Worlds are generated by the following partitions on $A$ :

- $w_{0}=\{\{\hat{a}\},\{\hat{b}\},\{\hat{c}\}\}$

- $w_{1}=\{\{\hat{a}\},\{\hat{b}, \hat{c}\}\}$

- $w_{2}=\{\{\hat{a}, \hat{b}\},\{\hat{c}\}\}$ 
- $w_{3}=\{\{\hat{a}, \hat{c}\},\{\hat{b}\}\}$

- $w_{4}=\{\{\hat{a}, \hat{b}, \hat{c}\}\}$

Object $a$ in the three-element model of classical mereology is located at the region $\{\hat{a}\}$, object $a+b$ at $\{\hat{a}, \hat{b}\}$, etc. To see that these worlds exhibit the right mereological structure, we need to check that in every case where ' $x$ is a proper part of $y$ ' is true in the model of mereology (i.e. every $x$ and $y$ connected by arrows), there are corresponding worlds in which $x$ is located in a subregion of $y$. Suppose we want to check whether it is correct to say ' $a+b$ is a proper part of $a+b+c$ ' at $w_{0}$. Paraphrasing, we need to check that there are worlds $w$ and $w^{\prime}$ such that they are P-duplicates of $w_{0}$, and $|a+b|_{w} \subseteq|a+b+c|_{w^{\prime}}$ and $|a+b|_{w} \neq|a+b+c|_{w^{\prime}}$. But $w_{2}$ and $w_{4}$ are respectively just such worlds. I leave it to the reader to confirm visually all remaining proper parthood claims.

Turning now to the fully general result: it can be shown that the paraphrases of mereological relations can be shown to satisfy all the axioms of classical extensional mereology.

(maI) Reflexivity: $x$ is part of $x$.

(ma2) Antisymmetry: if $x$ is part of $y$ and $y$ part of $x$, then $x$ is identical to $y$.

(ma3) Transitivity: if $x$ is part of $y$ and $y$ part of $z$, then $x$ is part of $z$.

(ma4) Strong Supplementation: if $x$ is not part of $y$, then there is some $z$ such that $z$ is part of $x$ and does not overlap $y$.

(mas) Unrestricted Composition: for any $x x$, there is some $y$ such that any $z$ overlaps $y$ iff $z$ overlaps one of $x x$.

I prove each axiom in turn. For (MAI), ' $x$ is part of $x$ ' is correct at $@$ iff there are worlds $w_{1}$ and $w_{2}$ such that they are P-duplicates of $@$, and $|x|_{w_{1}} \subseteq|x|_{w_{2}}$. Letting $w_{1}=w_{2},|x|_{w_{1}} \subseteq|x|_{w_{1}}$.

For (MA2), assume there are worlds $w_{1}$ and $w_{2}$ each P-duplicating @ such that $|x|_{w_{1}} \subseteq|y|_{w_{2}}$ and some worlds $w_{3}$ and $w_{4}$ each P-duplicating @ such that $|y|_{w_{3}} \subseteq|x|_{w_{4}}$. Since $w_{1}-w_{4}$ are all 
P-duplicates of @, they are P-duplicates of each other. In that case, $|x|_{w_{1}}=|x|_{w_{4}}$ and $|y|_{w_{2}}=|y|_{w_{3}}$. Substituting, we have it that $|x|_{w_{1}}=|y|_{w_{2}}$ and $|x|_{w_{4}}=|y|_{w_{3}}$, etc. So, they are located in the same region of the P-array at the relevant worlds. Does this mean they are 'identical'? Well, this depends on how one is to paraphrase talk about 'identity' in the context of mereology. According to the current paraphrase strategy, 'identity' ought to be interpreted as a transworld identity relation — or at least something identity-like: P-counterparthood. ${ }^{15}$

For (MA3), assume $w_{1}, w_{2}$ are P-duplicates of $@$, such that $|x|_{w_{1}} \subseteq|y|_{w_{2}}$ and $w_{3}$ and $w_{4}$ are P-duplicates of @ such that $|y|_{w_{3}} \subseteq|z|_{w_{4}}$. Since $w_{2}$ and $w_{3}$ are P-duplicates of @, they are Pduplicates of each other. In that case, $|y|_{w_{2}}=|y|_{w_{3}}$. Then by the transitivity of the subset relation on $A,|x|_{w_{1}} \subseteq|z|_{w_{3}}$.

To prove (MA4), assume that ' $x$ is not part of $y$ ' is correct. Paraphrasing the antecedent, there are no P-duplicate worlds in which $|x| \subseteq|y|$. So fix any two P-duplicate worlds $w_{1}$ and $w_{2}$ at which $\dot{x}$ and $\dot{y}$ exist; we know that $|x|_{w_{1}} \nsubseteq|y|_{w_{2}}$. Now let $|z|$ be the set of all points in $|x|_{w_{1}}$ not in $|y|_{w_{2}}$. Notice $|z| \subseteq|x|_{w_{1}}$ and the intersection of $|z|$ and $|y|_{w_{2}}$ is empty. Thus, ' $z$ is part of $x$ and does not overlap $y^{\prime}$ is correct, as required. But does $|z|$ exist at some P-duplicate world $w_{3}$ ? Yes, since every subset of the $\mathrm{P}$-array $A$ is a member of some possible partition on $A$, by the plenitude principle $|z|_{w_{3}}$ exists, as does $\dot{z}_{w_{3}}$.

To show that (MA5) holds, we want to show that ' $x x$ compose $y$ ' is correct for arbitrary $x x$. Paraphrasing, we want there to be some $w_{1}$ and $w_{2}$ such that $|y|_{w_{2}}=\bigcup|x x|_{w_{1}}$. Since $|x x|_{w_{1}}$ is a set of members of the partition $P$ on $A, \bigcup|x x|_{w_{1}}$ is a subset of $A$. But any subset of $A$ is a member of some possible partition $P^{\prime}$ on $A$. By the plenitude principle, there is some world $w_{2}$ in which $|y|$ exists. Set theory guarantees that for any $|z|,|z| \cap|x x|_{w_{1}}$ is non-empty iff $|z| \cap|y|_{w_{2}}$ is non-empty. Hence, ' $z$ overlaps $y$ iff $z$ overlaps one of the $\mathrm{xx}^{\prime}$ is correct, as required. And so we have it that composition, paraphrased as a relation between worlds, is unrestricted even though composition within a world never occurs.

\footnotetext{
${ }^{15}$ Cotnoir [3] shows how treating distinct but mutual parts as equivalents recaptures the full strength of the antisymmetry axiom. A similar approach could be used here.
} 
Now, classical mereology is consistent with there being no atoms at all. But on our above proposal, proper parthood chains will always bottom out in an 'atomic' world - the world in which objects are located according to the finest partition. But this is an inessential feature of the approach; we needn't have assumed that every possible partition will give acceptable locations for objects. Indeed, one might have thought that some regions (e.g. pointy ones) are not adequate locations for ordinary objects. We might, for instance, allow only certain types of regions as admissible locations for objects. ${ }^{[6]}$ One natural approach allows for the possibility of atomless gunk..$^{[7]}$

The key idea is to endow the set $A$ of spacetime points in a P-array with a topology..$^{18}$ Given a topological space, we can use notions of the interior (written ' $i(X)$ '), and closure (written ' $c(X)$ ') of a region. 19 We say a region $X$ is open iff it is identical with its interior, $i(X)=X$. A region is closed iff it is identical with its closure, $c(X)=X$. Furthermore, an open set $X$ is regular open iff $i(c(X))=X$; a closed set $X$ is regular closed iff $c(i(X))=X .^{20}$

If we allow only regular open regions as possible locations of objects, we can recapture 'gunk' using our paraphrase technique. We just need to tweak some of the definitions as follows:

partition* A set $P$ of non-empty sets is a partition* of $A$ if: (i) $c(\bigcup P)=A$; and (ii) the members of $P$ are regular open sets such that $p_{i} \cap p_{j}=\emptyset$ for any $p_{i}$ and $p_{j}$ in $P$ s.t. $i \neq j$ ).

p-counterpart* $\dot{x}_{w_{i}}$ and $\dot{y} y_{w_{j}}$ are $P$-counterparts ${ }^{*}$ iff $w_{i}$ and $w_{j}$ are P-duplicates, and $i\left(c\left(\bigcup|x x|_{w_{i}}\right)\right)=$ $i\left(c\left(\bigcup|y y|_{w_{j}}\right)\right)$.

\footnotetext{
${ }^{16}$ For more debate over possible 'receptacles' for objects, see Cartwright [2], Uzquiano [27], and Hudson [7, ch. 2]. The approach below is a variant of Tarski's [24] gunky models for mereology.

${ }^{17}$ Thanks to Agustìn Rayo for raising the question as to whether the approach can handle gunk.

${ }^{18} \mathrm{~A}$ topological space is any set $A$ together with a set of its subsets $\mathcal{O} \subseteq \wp(A)$ satisfying the following conditions:

I. $\emptyset$ and $A$ are both in $\mathcal{O}$

2. If $O \subseteq \mathcal{O}$, then $\bigcup O$ is in $\mathcal{O}$.

3. If $O_{1}, \ldots, O_{n}$ are in $\mathcal{O}$, then $O_{1} \cap \ldots \cap O_{n}$ is in $\mathcal{O}$.
}

The members of the collection $\mathcal{O}$ are the open sets. The complement of an open set is closed. We'll call the set of all closed sets $\mathcal{C}$.

${ }^{19}$ The interior of a set $X$ is the largest open set contained in $X: i(X)=\bigcup\{O \in \mathcal{O}: O \subseteq A\}$. The closure of a set $X$ is the smallest closed set containing $X: c(X)=\bigcap\{C \in \mathcal{C}: A \subseteq C\}$.

${ }^{20}$ Not all open sets are regular open, e.g. the complement of a point $x$ in Euclidean 3 -space with the standard topology; likewise not all closed sets are regular closed, e.g. the surface of a sphere in 3-space. 
We then plug in the starred versions of these into the remaining definitions. We can now show that MAI-MAs hold by generalising the above proofs. ${ }^{21}$ And importantly, we will vindicate the possibility of gunk, since for every object $x$ in some world $w_{i}$, there will be some P-duplicate world $w_{j}$ in which there is a $y$ such that $|y|_{w_{j}}$ is a proper subset of $|x|_{w_{i}}$. That's because every regular open region has another regular open as a proper subset. ${ }^{22}$ The fact that we can recover gunk is a marked advantage of this paraphrase strategy over virtually every other approach.23

\section{Recovering Ordinary Talk (Including Emergence!)}

I claim that nihilists are in no more difficult a position in paraphrasing ordinary talk about parthood and composition than are classical mereologists. We can extend this nihilistic interpretation of mereology to a full-fledged paraphrase for all talk of ordinary material objects.

Consider a sentence like: "The table is brown." Now, either there is a table in the actual world or there is not. If there is, then according to the above view, tables are heterogeneous extended simples and can have properties like being brown. Here we can simply co-opt the Ehring-McDaniel REGIONAL INSTANTIATION definition above: the table is brown at $r$ iff the table exemplifies browntropes existing at $r$. In this case, then, there is no need for paraphrase. If there are no actual tables, but merely particles arranged-table-wise, then by the plenitude principle on worlds there is some P-duplicate world to the actual world in which there really are tables with properties like being brown. And so, in correctly saying that the 'the table is brown', we are actually saying something about a specific region of our world that would be literally true at the very same region at the relevant

\footnotetext{
${ }^{21}$ For MA4: we cannot in general use $|x| \backslash|y|$ as our witness for $z$ in MA4, since $|x| \backslash|y|$ is not always regular open when $|x|$ and $|y|$ are. Example: take the regular open intervals $|x|=(0,1)$ and $|y|=(.5,1)$; then $|x| \backslash|y|=(0, .5]$ which is not regular open $-i(c((0, .5])=(0, .5)$. So, we need a different witness. Fortunately, $|x| \backslash c(|y|)$ will do, as it is always regular open, and disjoint from $|x|$.

For MA 5: we just need to show that for any non-empty subset $S$ of locations, $|z| \cap|y| \neq \emptyset$ iff $|x| \in S$ and $|z| \cap|x| \neq \emptyset$. We cannot in general use $|y|=\bigcup S$, since it's not always the case that unions of regular open sets are regular open. Example: $(0, .5) \cup(.5,1)$ is not regular open even if each of them is. However, if we let $|y|=i(c(\bigcup S))$, we have the result.

${ }^{22}$ For example, imagine a sphere with radius I without its surface. Now, there are uncountably many more surfaceless spheres with smaller radii contained in it. Is the centre point of the sphere in the domain? No. There are no point-sized regions, since they any such region will be closed and so not an admissible location.

${ }^{23}$ Although, see Williams [3 I] for a different attempt to simulate gunk.
} 
duplicate world.

More explicitly:

instantiation ' $x$ is $F$ at $r$ ' is correct at $w_{0}$ iff there is a world $w_{1}$ P-duplicating $w_{0}$ in which and $r \subseteq|x|_{w_{1}}$ and an $F$-trope exists at $r$.

In the special case where $\dot{x}$ exists at $w_{0}$, we can allow $w_{0}=w_{1}$. I allow $r$ to be a subset of the location of $\dot{x}$ simply because I want to allow for extended objects to instantiate properties in proper subregions of their locations (after all, they might be heterogeneous). In the case of a completely brown table, $r$ might well be identical to $|x|_{w_{1}}$. We thus get a very simple account of what it is for there to be 'some particles arranged table-wise': particles are arranged table-wise just if they are P-counterparts to a table!

The above approach raises a natural question: what sorts of properties can these material objects have? The answer given so far: whichever properties it inherits from the tropes at the relevant regions of the P-array. But how could such tropes determine all the properties of every object occupying that region? For example, consider again our simple table, and suppose it is heavy. Do properties like being heavy exist? Could being heavy be determined by perfectly resembling (mass) tropes? If so, the view appears to be committed to a version of HUMEAN SUPERVENIENCE, at least insofar as the properties had by macro-objects are completely determined by the properties (in this case, tropes) associated with the points they occupy.

I think this is merely an artefact of the Ehring-McDaniel account of properties; it may not be the case for the other accounts of property instantiation compatible with heterogeneous extended simples discussed in $\$$ I. But even still, the Ehring-McDaniel approach could be revised to allow for failures of the relevant version of HUMEAN SUPERVENIENCE. There might be emergent properties of objects that are not determined by associated tropes. To allow for this, we would need to supplement

\footnotetext{
${ }^{24}$ Thanks to an anonymous referee for raising this question. On the other hand, the proposal obviously flouts HUMEAN SUPERVENIENCE in a different way. A typical way of stating the thesis is: no two worlds differ at all without some corresponding difference in the arrangement of qualities. But worlds on this proposal clearly differ by virtue of the objects that exist there, even though their arrangement of qualities (i.e. P-array) stays the same.
} 
our earlier definition of a world from triples $w=\langle A, P, D\rangle$ to quadruples $w=\langle A, P, D, E\rangle$ where $E$ here is a set of emergent properties whose bearers are members of the domain. ${ }^{25}$

If worlds of this sort are allowed, then sentences like 'The table is heavy' can be paraphrased, even if being heavy is an emergent property. On this view, 'The table is heavy' is correct iff there is some world P-duplicating ours in which there is a table (in $D$ ) and it bears the property of being heavy (in $E$ ).

\section{Concluding Remarks}

Mereological nihilists are faced with a difficult challenge: explaining ordinary talk about material objects and their mereological relations. I have put forward a new paraphrase - the counterpart strategy — that has distinct advantages over its rivals. The main assumption is a commitment to a liberal view of the nature of simples; the nihilist must be willing to accept the possibility of heterogeneous extended simples. As a result, the mereological nihilist can accept that her position is metaphysically necessary, and yet can endorse the correctness of all the claims of classical mereology, including paraphrases involving gunky worlds. Nihilists, then, are in exactly the same position as the classical mereologist when it comes to explaining talk about ordinary objects, but without the additional ontology. In addition, the nihilist can paraphrase lots of other ordinary claims as well, potentially including claims about macro-sized objects that have emergent properties. These advantages are significant enough to make the proposal worthy of serious consideration. Whether the counterpart paraphrase strategy faces other problems remains to be seen. ${ }^{20}$

\footnotetext{
${ }^{25}$ Thanks to Jessica Wilson and Benj Hellie for pressing me to get clearer on this point.

${ }^{26}$ Thanks to Don Baxter, Björn Brodowski, Tim Elder, Gerry Hough, Aidan McGlynn, David Nicolas, and four anonymous referees for helpful comments on earlier versions of this paper. Thanks also to audiences at the University of Toronto (especially Imogen Dickie, Benj Hellie, Jessica Wilson, and Byeong-Uk Yi), the Metaphysical Indeterminacy Workshop at the University of Leeds (especially Elizabeth Barnes, Ross Cameron, Agustìn Rayo, Jason Turner, and Robbie Williams), the University of St Andrews (especially Derek Ball, Ephraim Glick, Patrick Greenough, Katherine Hawley, and Simon Prosser), and the petaf Modality Workshop at Stockholm University (especially Fabrice Correia, Andy Egan, Dan López de Sa, Peter Pagin, Sven Rosenkrantz, and Elia Zardini) for stimulating discussion of these ideas.
} 


\section{References}

[I] Cameron, R. How to have a radically minimal ontology. Philosophical Studies is (2010), 249-64.

[2] Cartwright, R. Scattered objects. In Analysis and Metaphysics, K. Lehrer, Ed. Reidel, Dordrecht, I975, pp. I53-I7I.

[3] Cotnoir, A. J. Anti-symmetry and non-extensional mereology. The Philosophical Quarterly 60,239 (2010), 396-405.

[4] Dorr, C., And Rosen, G. Composition as a fiction. In The Blackwell Guide to Metaphysics, R. M. Gale, Ed. Blackwell, 2002, pp. I 5 I-74.

[5] Ehring, D. Lewis, temporary intrinsics, and momentary tropes. Analysis 57 (I997), 254-258.

[6] Hazen, A., and Humberstone, L. Similarity relations and the preservation of solidity. Journal of Logic, Language, and Information I3 (2004), 25-46.

[7] Hudson, H. The Metaphysics of Hyperspace. Oxford University Press, 2006.

[8] LewIS, D. Tensing the copula. Mind III (2002), I-I 4.

[9] Markosian, N. Simples. Australasian Journal of Philosophy 76 (I 998), 2 I 3-26.

[io] Markosian, N. Simples, stuff, and simple people. The Monist 87 (2004), 405-28.

[I I] McDaniel, K. Extended simples. Philosophical Studies 133 (2007), I 3 I-4I.

[i2] McDaniel, K. Extended simples and qualitative heterogeneity. The Philosophical Quarterly 59 (2009), 325-33I.

[13] Merricks, T. Objects and Persons. Clarendon, Oxford, $200 \mathrm{I}$.

[i4] O'Leary-Hawthorne, J., and Cortens, A. Towards ontological nihilism. Philosophical Studies 79 (I995), I43-I65.

[i 5] Parsons, J. Distributional properties. In Lewisian Themes, F. Jackson and G. Priest, Eds. Oxford University Press, 2004, pp. I73-80.

[i6] Rea, M. How to be an eleatic monist. Philosophical Perspectives IS (200I), I 29-I 5 I.

[i7] Schaffer, J. From nihilism to monism. Australasian Journal of Philosophy 85 (2007), I75-9I.

[i8] Schwarz, W. Parts and counterparts. http://www.umsu.de/papers/parts.pdf, 2005.

[ig] Sider, T. Against parthood. (ms.).

[20] Sider, T. All the world's a stage. Australasian Journal of Philosophy 74 (I996), 433-53. 
[2 I] Sider, T. Monism and statespace structure. In Being: Developments in Contemporary Metaphysics, R. L. Poidevin, Ed. Cambridge University Press, 2008, pp. I 29-I 50.

[22] Simons, P. Extended simples: a third way between atoms and gunk. The Monist 87 (2004), $37 \mathrm{I}-384$.

[23] Spencer, J. A tale of two simples. Philosophical Studies I48, 2 (20 Io).

[24] Tarski, A. Foundations of the geometry of solids. In Logic, Semantics, Metamathematics, J. Corcoran, Ed. Hackett, 1956.

[25] Turner, J. Ontological nihilism. In Oxford Studies in Metaphysics, D. Zimmerman and K. Bennett, Eds., vol. 6. Oxford University Press, 20 I I.

[26] Unger, P. There are no ordinary things. Synthese 4I (I979), I I7-54.

[27] Uzquiano, G. Receptacles. Philosophical Perspectives 20 (2006), 427-45 I.

[28] van Inwagen, P. The doctrine of arbitrary undetached parts. Pacific Philosophical Quarterly 62 (I98I), I $23-\mathrm{I} 37$.

[29] van Inwagen, P. Material Beings. Cornell University Press, I995.

[30] Wheeler, S. On that which is not. Synthese 4I 4I, 2 (I979).

[3 I] Williams, J. R. G. Illusions of gunk. Philosophical Perspectives 20, I (2006), 493-5 I3.

[32] Williams, J. R. G. Gavagai again. Synthese I64, 2 (2008), 235-259. 